\title{
Programming Methods for DNA-Based Reaction-Diffusion Systems
}

\author{
Keita $\mathrm{Abe}^{1} \cdot$ Satoshi Murata ${ }^{1}$
}

Received: 15 December 2019 / Accepted: 8 April 2020 / Published online: 11 May 2020

(c) The Author(s) 2020

\begin{abstract}
In this tutorial, recent development of pattern generation algorithms based on DNA computing will be overviewed. Natural pattern generation, especially in biological organisms, are often driven by spatio-temporal chemical reactions. Various reaction-diffusion systems have been proposed to generate artificial patterns out of DNA sequences. To program DNA reaction-diffusion systems, in addition to the design of the reaction, diffusion of each DNA species must be considered. This is realized by immobilizing or suppressing diffusion of DNA molecules in the reaction field. Here, several typical methods to build patterns by 1-D or 2-D reaction-diffusion systems are introduced and how to implement the system by DNA molecules is explained. The direction of future research and possible applications of this technology will be also discussed.
\end{abstract}

Keywords DNA computing $\cdot$ Reaction-Diffusion system $\cdot$ Hydrogel $\cdot$ DNA PEN toolbox

\section{Introduction}

Various patterns such as spirals, stripes, and spots are found in nature. Many of them are classified as dissipative structures, which appear in thermal, chemical, and other dissipative processes [1]. In particular, living organisms are known to form versatile patterns through complex biochemical processes [2]. For instance, skin cells synthesize different proteins which affect the interaction among the cells, resulting in a characteristic pattern on the skin. The mechanisms of biological pattern formation are not yet completely understood, however, a gene expression in the cell and

Keita Abe

abe@molbot.mech.tohoku.ac.jp

Satoshi Murata

murata@molbot.mech.tohoku.ac.jp

1 Department of Robotics, Graduate School of Engineering, Tohoku University, Sendai, Japan 
propagation of molecules among the cells, i.e. reaction and diffusion, are thought to be essential.

Biological pattern formations have been modelled using reaction-diffusion systems [3], described as a set of partial differential equations. For instance, chemical species $X$ and $Y$ react and produce $Z$, namely

$$
X+Y \rightarrow Z
$$

And the spatio-temporal behaviour of the system is modelled as follows;

$$
\begin{aligned}
& \frac{\partial}{\partial t}[X]=D \Delta[X]-k[X][Y], \\
& \frac{\partial}{\partial t}[Y]=D \Delta[Y]-k[X][Y], \\
& \frac{\partial}{\partial t}[Z]=D \Delta[Z]+k[X][Y],
\end{aligned}
$$

where $[X],[Y]$, and $[Z]$ represent the concentration of species $X, Y$, and $Z$, respectively, $D$ is the diffusion coefficient, and $k$ is the rate constant of the reaction.

The concentration change caused by diffusion is in proportion to $D$ and the spatial second derivative of the concentration. The diffusion coefficient $D$ is affected by the size of the molecule, temperature, and viscosity of the medium. In this example, the reaction term is a simple multiple of $k$ and concentrations of $X$ and $Y$. The spatio-temporal pattern formation process can be simulated by solving these equations.

The most famous example of a reaction-diffusion system was given by Turing [4]. By choosing appropriate values for the diffusion coefficient and the reaction rate, the system is able to reproduce various patterns such as fish scales, animal furs, and sea shells [5-7].

In addition to these analytic research, researchers made an artificial reaction-diffusion system in a test tube. The Belousov-Zhabotinsky (BZ) reaction is a typical example [8,9], which exhibits oscillatory behaviour between two metal ions via redox reactions. In a $2-\mathrm{D}$ reaction field, the reaction produces spatio-temporal patterns such as a spiral. Theoretical studies have revealed relationships between the geometric properties of formed patterns and chemical parameters in the system; however, it is difficult to manipulate the pattern for specific purposes $[10,11]$.

\section{Reaction Programming Using Synthetic DNA}

To program a reaction-diffusion system with the desired pattern formation capability, it is required to implement them as a chemical reaction. For this purpose, synthetic DNA has a great potential.

DNA is composed of a backbone polymer attached with bases. The backbone is a simple repeat of sugar and phosphate groups, and one of the four bases [adenine (A), guanine $(\mathrm{G})$, cytosine $(\mathrm{C})$, or thymine $(\mathrm{T})]$ is attached to each sugar molecule. The sequence of the bases stores genetic information of living organisms, but our primary interest is not in DNA's biological functionalities, rather, we focus on DNA's 


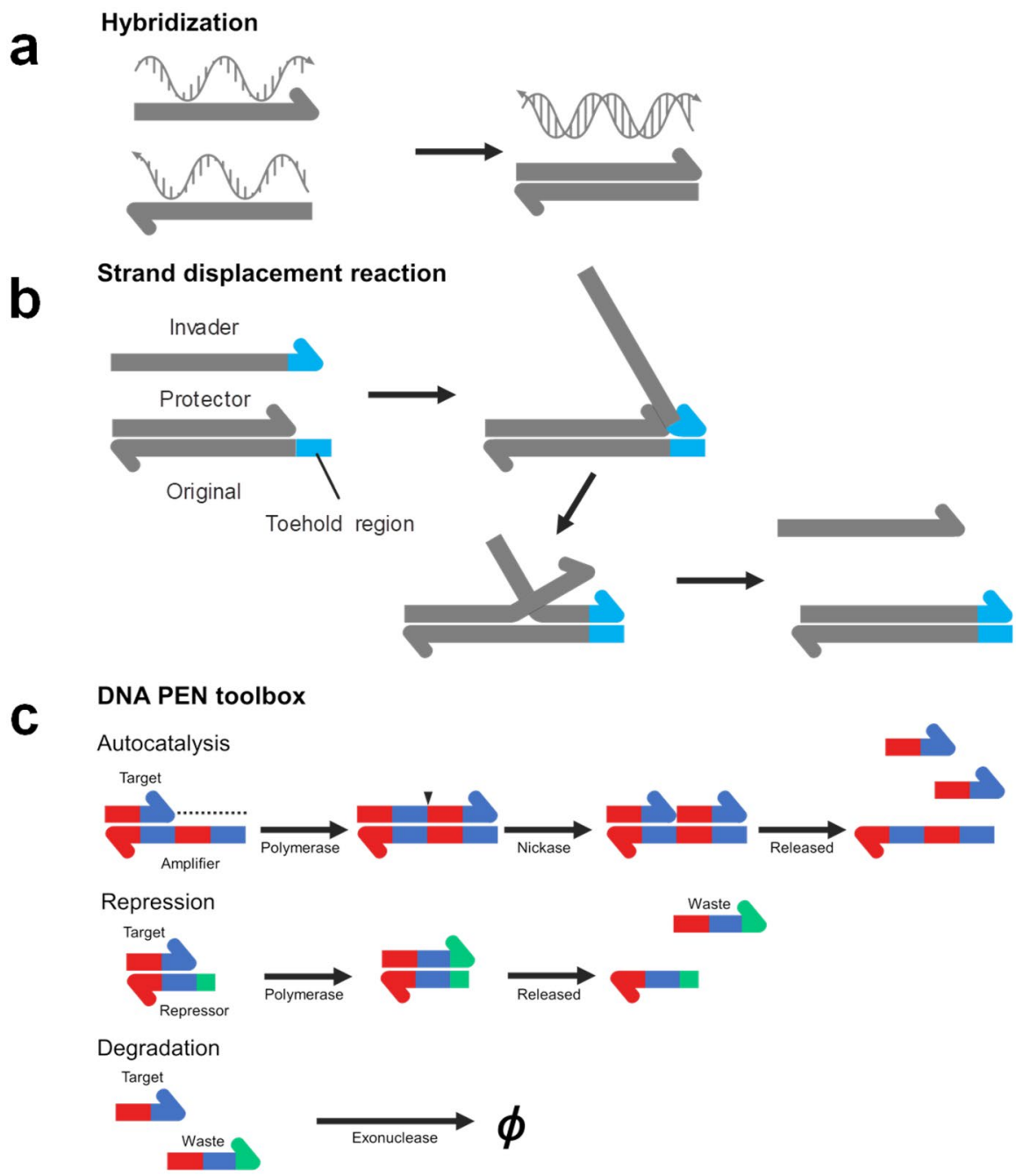

Fig. 1 Basic DNA reactions. a A process of hybridization. Two DNA strands hybridize each other and form a double-stranded DNA. Each arrow represents a single strand of DNA. b Toehold-mediated strand displacement reaction. The toehold regions initiate the process by allowing Invader to hybridize with Original. After the binding of Invader at the toehold, branch migration of invading domain results in the displacement of Protector. c DNA PEN toolbox. In the autocatalysis process, Target is amplified by DNA polymerase and nickase, using Amplifier as a catalyst. After the nicking, the original and produced Target strands are spontaneously released when the temperature is set around the melting temperature (melting temperature is the temperature at which 50\% of DNA strands can maintainhybridization) between Target and Amplifier strands. In the repression process, Target is elongated and it becomes Waste which is released by thermal fluctuation. Waste cannot be extended by polymerase, because its $3^{\prime}$ end does not hybridize with Amplifier. Both Target and Waste are degraded by exonuclease.

specific properties based on its complementarity. It is defined as a property that only the combination of $\mathrm{A} / \mathrm{T}$ and $\mathrm{G} / \mathrm{C}$ forms a pair (Watson-Crick base pairing) [12]. A pair of DNA strands with complementary base sequences forms a double helix (i.e. 
hybridize) (Fig. 1a). Thermodynamic properties of DNA have been so well studied that it is possible to predict the specific parts of sequences that hybridize in a pool of various DNA strands. (This is called the secondary structure prediction problem ${ }^{1}$ ). By solving the inverse problem of the structure prediction, it is able to design a set of sequences which take a desired secondary structure [14-19].

A double helix made of a complementary pair of DNA is the most stable structure in an aqueous environment. However, if there is a single-stranded region called a toehold (Fig. 1b), it is possible to replace one of the DNA strands in the double helix [20]. This reaction is called toehold-mediated strand displacement, and is often utilized in the design of DNA reaction systems. A cascade of the strand displacement reactions has been applied to various DNA computing systems [21-25].

Oscillatory or cyclic reactions are difficult to realize in this way, because free energy difference of the strand displacement reaction is very small, since the driving force of the strand displacement reaction is only due to the hydrogen bond formation at the toehold region. DNA enzymes capable of synthesizing and degrading new DNA strands solve this problem.

The DNA PEN toolbox is one of the most well-established approaches to build enzymatic DNA reaction system with dynamic properties. In the PEN toolbox, Polymerase elongates DNA, Nickase cleaves DNA at a specific sequence, and Exonuclease non-specifically degrades DNA. These three enzymes are combined to build arbitrary reaction dynamics (Fig. 1c) [26-28].

In the following sections, we review reaction-diffusion systems using DNA computing technology. All of them but the system in [29] were implemented in laboratories, some of them being supported by simulation. In [29], the programmability of the reaction-diffusion system was examined in silico only.

\section{A DNA Reaction-Diffusion System in Hydrogel}

Well-designed DNA reactions combined with diffusion of DNA is a promising way to program artificial reaction-diffusion systems. For predicting and designing such DNA reactions, several online services such as DINAMelt [30], NUPACK [31], and Visual DSD [32] are useful. Especially, Visual DSD is capable of solving partial differential equations [33], which is essential to predict the spatial behavior of the reaction-diffusion system.

In [29], the authors examine the programmability of DNA reaction-diffusion systems by a computer simulation. The goal of this research is to program a system generating a desired pattern. The system is hierarchically built using primitive reaction modules such as duplication, overlap, extraction, inversion, and blur. By combining these modules under given boundary conditions, the desired pattern is constructed. As this system requires many species of DNA, experimental implementation is not realistic in its present form.

\footnotetext{
${ }^{1}$ It is possible to predict the secondary structure made of various DNA strands under the assumption that the structure does not contain pseudoknots [13].
} 
a

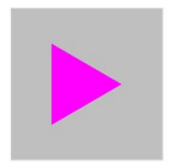

UV light pattern

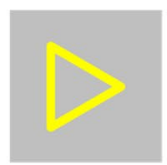

Edge detection

b

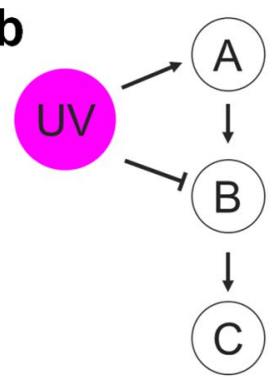

Activation cascade
C
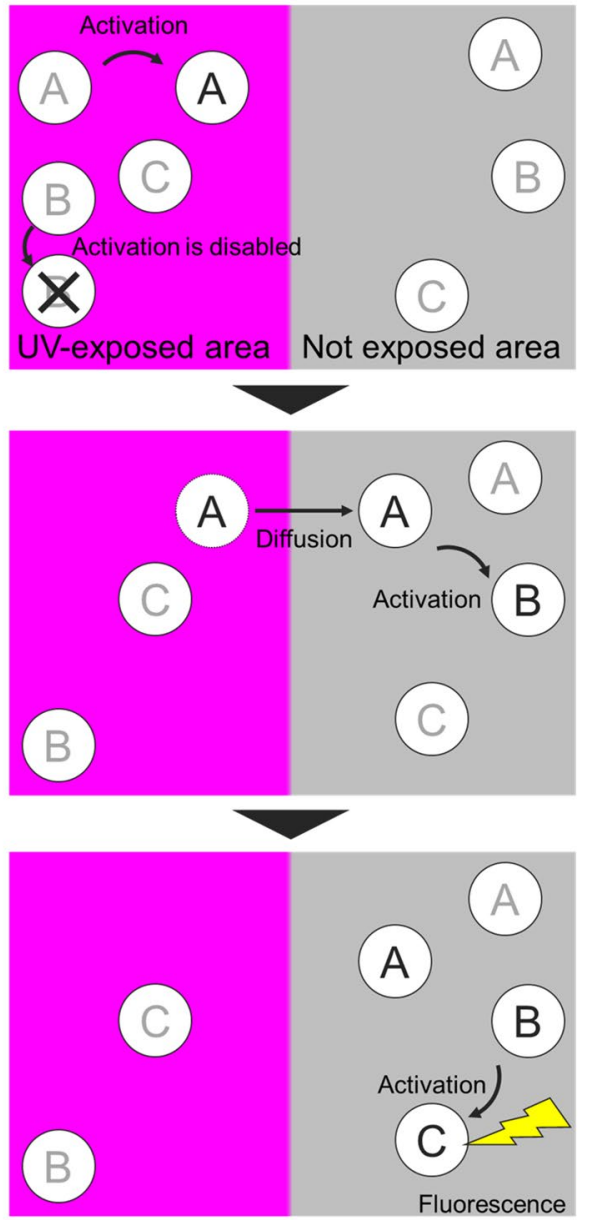

Activation process on the boundary

Fig. 2 Edge detection system. a Edge detection of UV-irradiated area. b UV light activates A and disables activation of B. $\mathbf{c}$ In the UV-exposed area, A is activated and the activation of B is disabled. The activated A diffuses into the region not exposed to UV, and activates B there. The activated B activates $\mathrm{C}$, and the result is visualized by fluorescence. Adapted from [35]

The order of the reaction rate and diffusion coefficient must be considered in the design of reaction-diffusion systems. For instance, in the case of a strand displacement of $50 \mathrm{nt}$ DNA at a concentration of $1 \mu \mathrm{M}$, its diffusion coefficient is about 40 $\mu \mathrm{m}^{2} \mathrm{~s}^{-1}$ [34], while its reaction rate is $3 \times 10^{6} \mathrm{M}^{-1} \mathrm{~s}^{-1}$ [16]. Therefore, the diffusion term $\left(40 \mu \mathrm{M} \mathrm{s}^{-1}\right)$ is ten times larger than the reaction term $\left(3 \mu \mathrm{M} \mathrm{s}^{-1}\right)$. In general, diffusion is much faster than the hybridization reaction. The strand displacement reaction is even slower. To produce spatio-temporal patterns, these terms must be balanced. 
Fig. 3 Reaction-diffusion system using reservoirs. a Schematics of the system. Reservoir 1 contains Initiator, Recapturer, and Reporter; Reservoir 2 contains a Source, Recapturer, and Reporter; and the hydrogel contains Recapturer and Reporter. b) DNA reaction in the system. Release: initiator and Source produce Waste 1 and Output by strand displacement. Recapture: output reacts with Recapturer and produces Waste 2 and 3 in a slow irreversible process. Reporting: output reacts with Reporter. Black and yellow circles represent a quencher and a fluorescent molecule, respectively. Adapted from [36]

One approach to solve this is to build a reaction-diffusion system in hydrogel medium to suppress diffusion [35-38]. A system for 2D edge detection in polyacrylamide gel is a good example (Fig. 2) [35]. It can detect the circumference of UV-exposed area on the gel. The outline of the mechanism is as follows. The system employs three DNA species A, B, and C; each of them has an active and an inactive state, and all of them are "inactive" at the beginning. An activation cascade occurs in three steps. First, UV irradiation activates A and disables the activation of B. Second, the activated $\mathrm{A}$ diffuses into the non-irradiated area and activates $\mathrm{B}$. Third, the activated B activates $\mathrm{C}$ to emit fluorescence. Since $\mathrm{C}$ is activated only around the circumference of the area, the overall reaction works as an edge detector. Special photocleavable DNA is used for implementation of the system. The obtained edge pattern cannot be maintained against dissipation.

To keep formed pattern in the medium, we need to compensate lost molecules by diffusion. For this purpose, some systems adopt reservoirs of DNA. Zenk et al. use reservoirs as a part of boundary condition, where the concentration of DNA in it is maintained by constant replacement of the solution (Fig. 3) [36]. The pattern is visualized by three reactions: release, recapture, and reporting. Initiator and Source are diffused into hydrogel from Reservoir 1 and 2, react there, and release Output by strand displacement (Release). It is visualized by fluorescence in the reversible reaction (Reporting) then slowly consumed to make Waste (Recapture). Without supply, the obtained pattern gradually decays. By supplying DNA from the reservoirs, the generation of Output DNA continues, and thus the pattern is maintained.

\section{A Reaction-Diffusion System Using an Enzymatic Reaction}

The concentration of DNA can also be maintained by balancing enzymatic synthesis and degradation. In $[39,40]$, the DNA PEN toolbox is used to make a French flag pattern of DNA molecules (Fig. 4). A reactions among repressor, amplifier, and target are designed, in which either autocatalysis or repression is dominant, depending on the concentration of the reference. Gradient of amplifier concentration is used as a reference to make a pattern that divides the whole field into three regions. Target (red) is amplified when its concentration is lower than the reference and decomposed when it is higher than the reference (Fig. 4a). It is also possible to design an antagonistic reaction system where the production of one species [Target 1 (red)] suppresses the other [Target 2 (blue)] and vice versa (Fig. 4b). The resultant French flag pattern is maintained with this mechanism.

Not only stationary patterns, but also dynamic (ever-changing) patterns can be generated using a chemical oscillator. In [41, 42], a propagating wave pattern is 
a
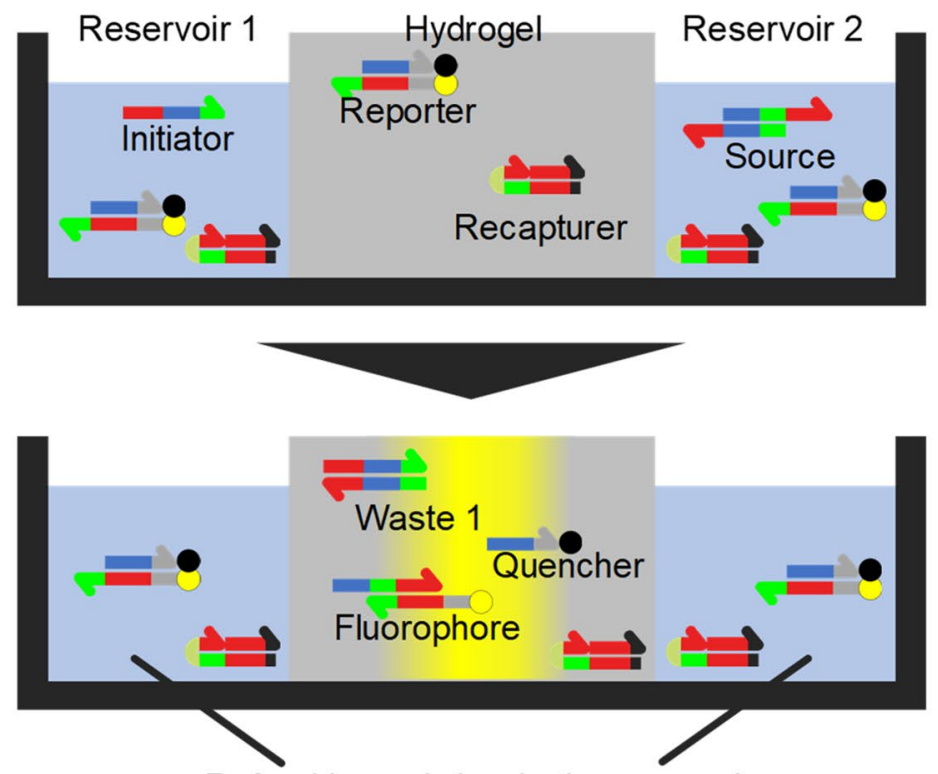

Refreshing solution in the reservoirs

b

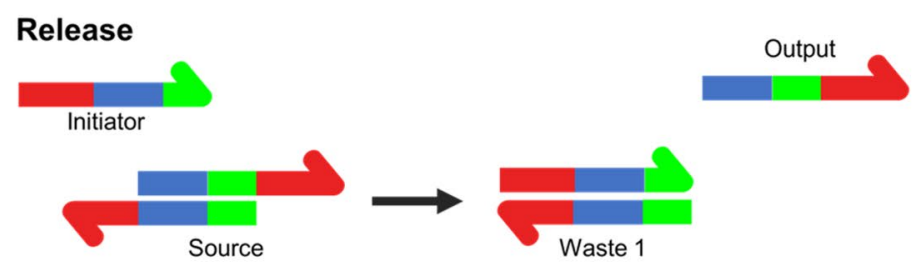

\section{Recapture}

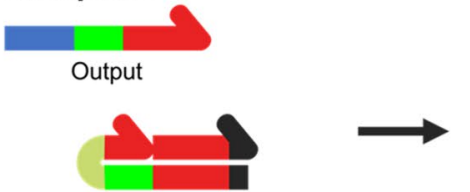

Recapturer

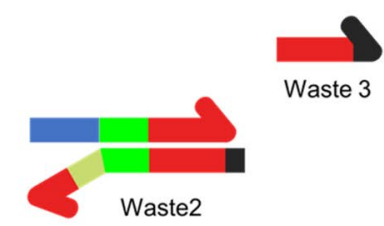

\section{Reporting}

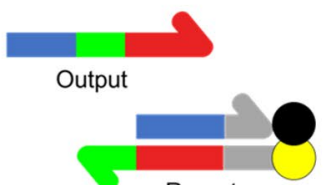

Reporter
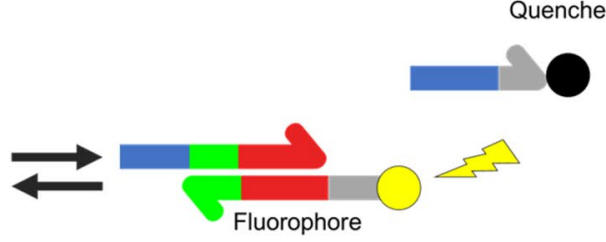

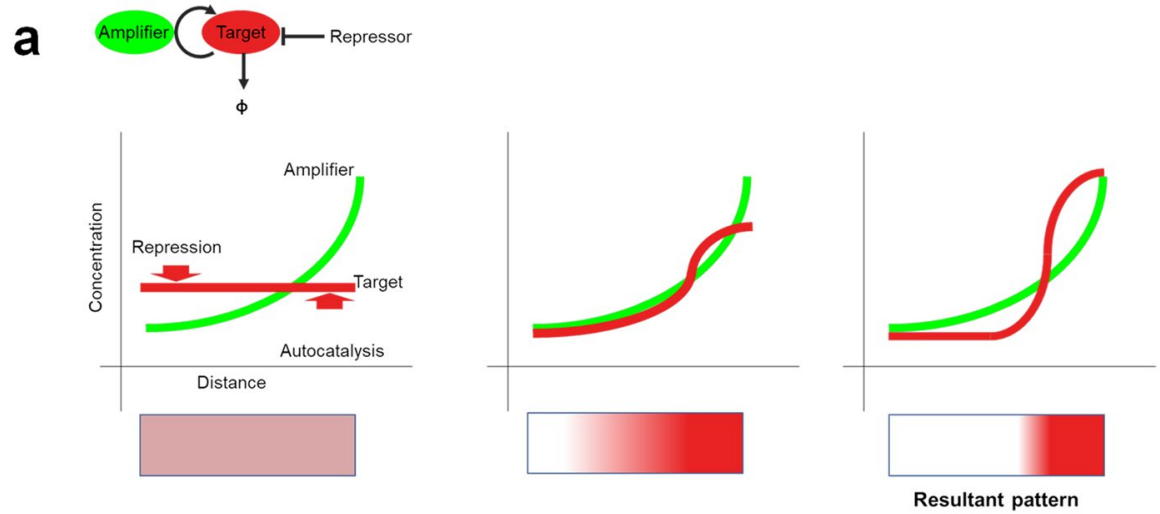

b
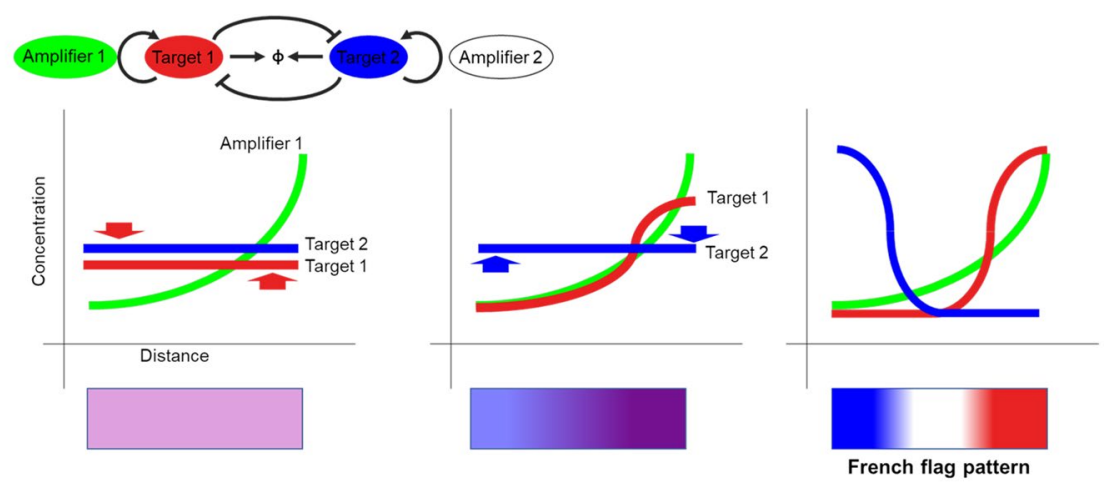

Fig. 4 French flag pattern formation. a Initial distribution of Target (red) and Reference molecule (green). When Amplifier has a concentration gradient, repression is dominant in the left side and autocatalysis is dominant in the right side. b Target 1 (red) is amplified by using Amplifier 1 (green). It inhibits Target 2 (blue), and Target 2 inhibits Target 1 . As a result, the distribution of Target 1 and 2 forms the French flag pattern. Adapted from [39]

produced by using a reaction system derived from the predator-prey model (Fig. 5). Under certain conditions, the population oscillates in a constant cycle. Namely, the number of prey increases by using grass as a catalyst. On the other hand, the number of predators increases by prey. This process increases predator, while it decreases prey. A fluctuation in the number of prey is transmitted to predator with a certain time delay, which generates an oscillation.

Here, DNA strand representing prey is duplicated on grass strand by polymerization followed by a nicking reaction (Fig. 5a). Prey is also elongated on predator, which transforms prey to predator. Exonuclease decomposes both of them. The overall reaction system combining these reactions exhibits wave propagation in $2 \mathrm{D}$ reaction field (Fig. 5b). 


\section{a}

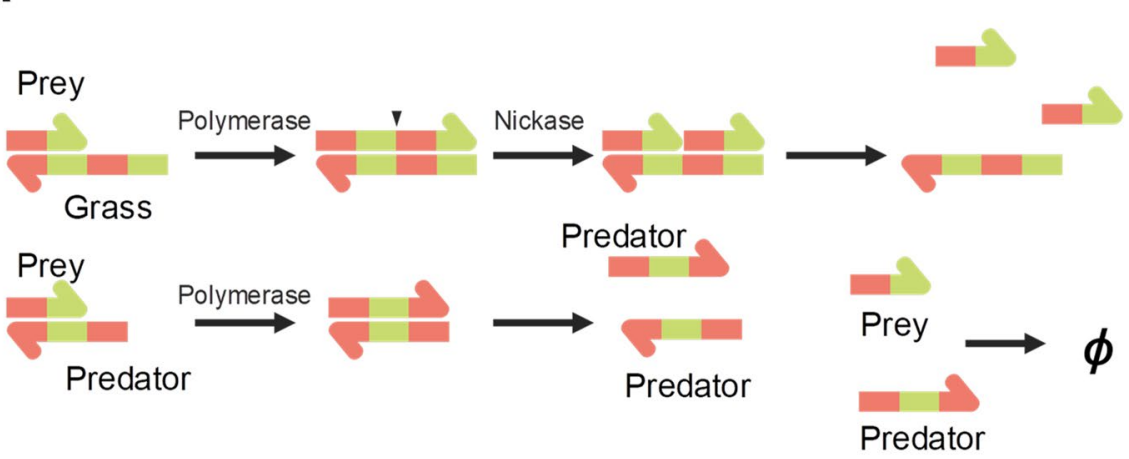

b
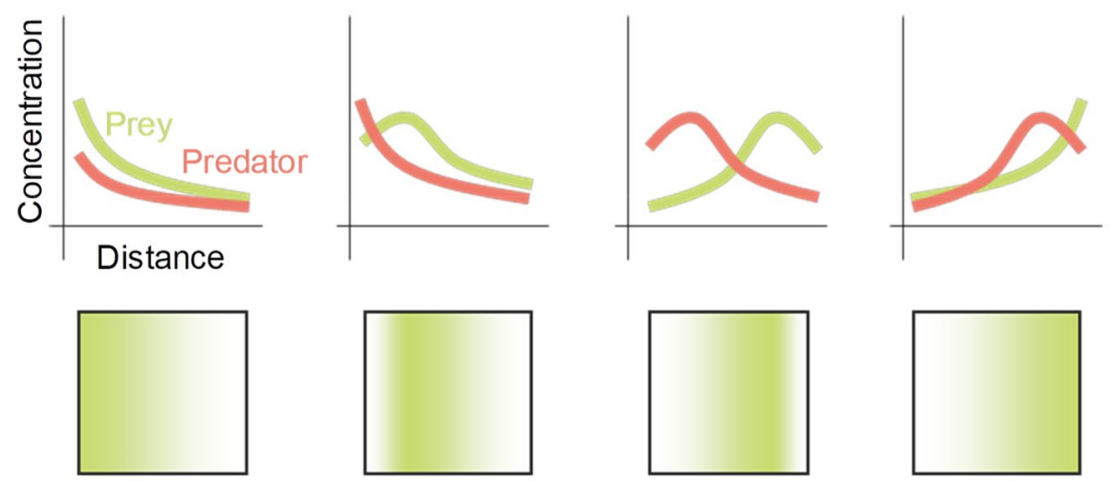

Prey distribution

\section{Time}

Fig. 5 Wave pattern formation using enzymatic reactions. a Mechanism of the prey-predator model using DNA. Prey is amplified by grass, and predator increases by prey. Both predator and prey are degraded by exonuclease. b Distribution of prey and predator. The wave propagates from left to right. Adapted from [41]

\section{Pattern Formation with Programmed Diffusion}

In the previous section, several DNA-based reaction-diffusion systems have been introduced. These systems are designed on the premise that all DNA species diffuse in the medium. Here, we introduce approaches to tune diffusion speed of individual DNA species, which enables us to extend the design ability of reaction-diffusion systems. 


\section{a}

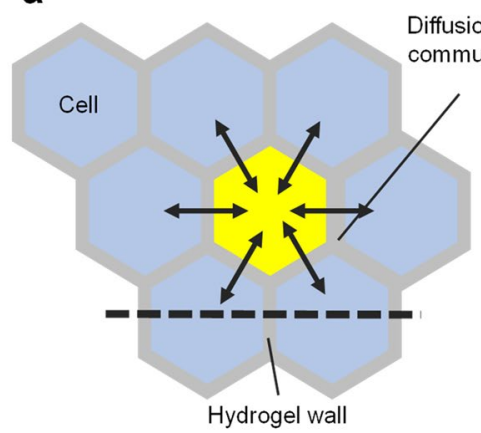

b

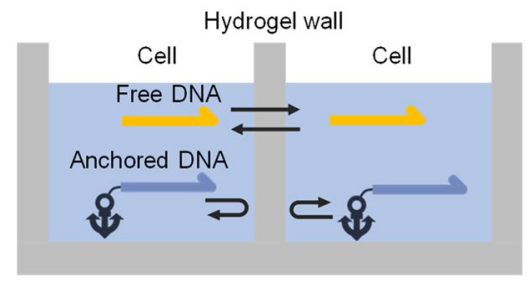

Fig. 6 Gellular automata using anchored DNA. a Each hexagon represents a cell separated by hydrogel walls. Cells contain DNA logic gate and DNA signals propagate to the neighbouring cells. b Side view. Free DNA strands can pass through the hydrogel wall and propagate to neighbour cells, but the anchored DNAs, which cannot diffuse in the hydrogel, stays inside the cell. Adapted from [45]

The first example is DNA immobilization developed in our group. The Stokes-Einstein equation tells us that larger molecules diffuse slower. When a DNA is anchored to a polymer, it becomes non-permeable through the hydrogel wall. Moreover, by using strand displacement, it is possible to attach and detach a molecule from the anchored DNA, meaning that diffusion can be turned on or off by another DNA.

We have applied the DNA immobilization to realize the concept of gellular automaton [43-45]. Gellular automaton is a materialized cellular automaton consisting of an array of cellular compartments containing a solution of molecular computers (Fig. 6a). The compartment is made of a hydrogel wall, mimicking the cell wall, which allows only small molecules to pass through [45]. In the gellular automata, we chose the property of the gel such that free DNA which is not anchored can diffuse through it (Fig. 6b). We use an acrydite-modified DNA copolymerized with acrylamide to make an anchored DNA. To represent the state transition of cellular automata, a DNA logic gate is immobilized in a cell, and their outputs pass through the wall to realize intercellular communication.

The second approach is a direct control of diffusion. This method achieves sequence-specific tuning of the diffusion speed by using reversible toehold exchange reaction. Both Target and Competitor are designed to hybridize with Anchor (Fig. 7a). The net diffusion coefficient of Target can be tuned by the concentration of Competitor, because the equilibrium of the reaction depends on the concentrations of those reactants. Experiment shows that the diffusion coefficient can be modulated by a factor of 6 [46]. We also demonstrated sequence-specific modulation among orthogonal DNA sequences.

The last example is pattern formation utilizing diffusion modulation. By combining a logic gate, anchoring, and diffusion modulation, we are able to program both reaction and diffusion [38]. In the experiment, an AND gate outputs fluorescence only when it receives both Input A and B using associated toehold activation [21]. The gate is anchored to the gel matrix and is uniformly distributed over 
a

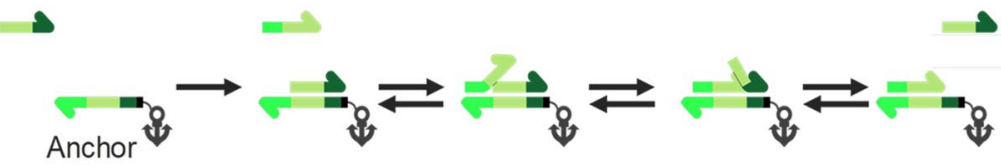

b
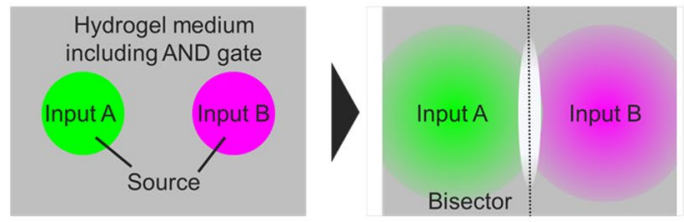

C
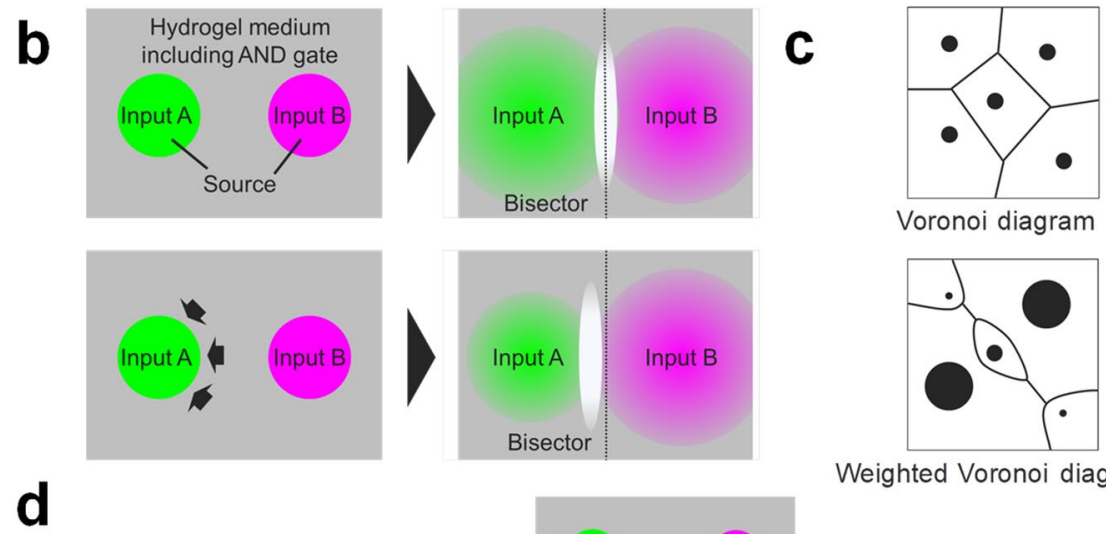

Bisector

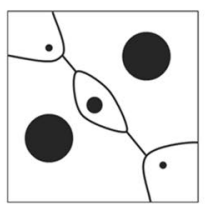

Weighted Voronoi diagram

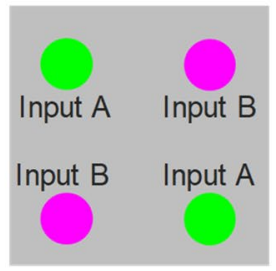

Initial pattern
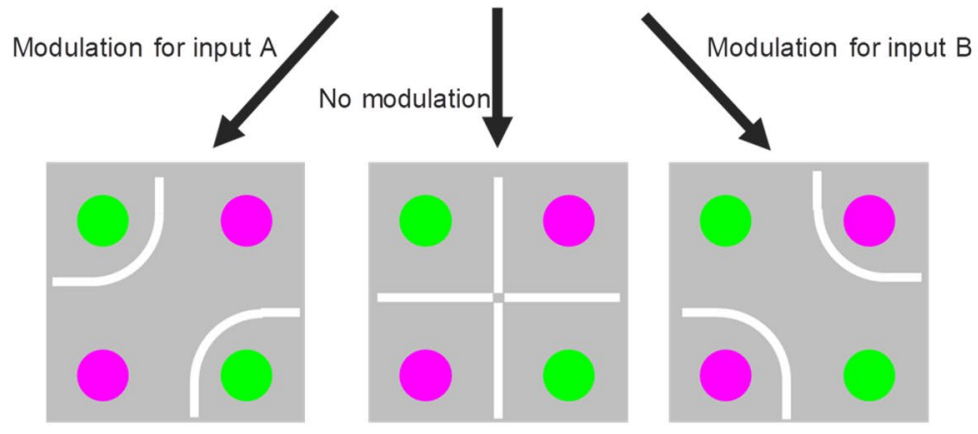

Resultant patterns

Fig. 7 Pattern formation using diffusion modulation. a Mechanism of diffusion modulation. Target and Competitor competitively hybridize with Anchor. When Target hybridizes with Anchor, Competitor displaces Target in a reversible way. b Schematic of bisector pattern formation. Input A and B diffuse from their sources and form a bisector at an equal distance from the sources. When diffusion of Input A is supressed, the position of bisector shifts to the left. $\mathbf{c}$ Schematic of Voronoi and weighted Voronoi diagrams. d Weighted Voronoi pattern formation by using diffusion modulation. Adapted from [38]

the gel. Input A and B are diffused from source points located at the corner of the reaction field. As a result, fluorescence was observed on the bisection between the sources when no modulation was applied (Fig. 7b). The position of the bisector is 
shifted by the diffusion modulation. Anchor for Input A is distributed in the gel, and when there is no Competitor, Input A can be trapped by nearby Anchor, therefore its diffusion is strongly suppressed. In this case, the bisector appears near source A. If Competitor is added, the suppression become smaller, and the position of the bisector shifts toward source B depending on its concentration. This process is verified by experiments and was consistent with the simulation (Fig. 7c). We have also performed experiments on a field containing two pairs of sources. When there are no Competitor and Anchor, the system forms a cross-shaped bisector at the center. When the diffusion modulation is applied, it forms two curves that correspond to the weighted Voronoi diagram (Fig. 7d).

\section{Future Issues}

As seen in this tutorial, various reaction-diffusion systems have been proposed so far. In those systems, the programmability of DNA has greatly increased the freedom of design. Much research is still underway, and it seems that more advanced pattern formation will be possible in the near future. In this section, several directions for future research is discussed.

One obvious direction is to combine existing methods to manipulate reaction and/ or diffusion. It has already been proved that the DNA PEN toolbox and the anchoring mechanism can coexist in the same hydrogel [47]. For instance, a combination of DNA enzymes and diffusion modulation will enable us to design more elaborate patterns which exhibit temporal development.

The second direction is to realize a hierarchical system. By mimicking the pattern formation in embryogenesis [48, 49], a multistage DNA reaction diffusion system can be designed. For instance, we can utilize the formed pattern as the initial condition for the next reaction stage, which generates more complex patterns.

The third direction is to scale up the system. Thanks to the rapid development of DNA synthesis technology, large amounts of synthetic DNA are now available at low cost. This would enable us to build a system at centimeter scale or even larger for various applications.

The fourth direction is functionalization of the formed patterns. Although DNA itself has almost no chemical function, it can be functionalized by various kinds of modification. For instance, using DNA segments, coding proteins is a promising approach to functionalize the formed pattern [50]. A DNA sequence which recognizes a specific molecule called an aptamer is also useful. A hydrogel with such a recognition ability will produce a system responsive to various chemical signals. This will be useful for tissue engineering [51].

The last but not the least significant example is to utilize the physical properties of DNA hydrogel. Recently, DNA hydrogel that swells and shrinks in response to DNA signal has been reported [52-55]. By using this method, the generated pattern on a gel can work as a movable object or an actuator.

Controlled gelation of various synthetic polymers have been reported [55-59]. Reaction-diffusion systems can be built by using these polymers. DNA hydrogel 
is much more powerful than these polymer materials with no programmability. For instance, gelation/melting of DNA can be controlled by a chemical signal such as a short DNA segment or physical stimuli such as UV irradiation [60].

Thanks to the rapid price down in the synthetic DNA, functionalized DNA gels combined with programmed reaction-diffusion will open various new applications. It also gives a foundation to build robots made of chemical materials [61].

Acknowledgements This tutorial was supported by JSPS KAKENHI (Grants-in-Aid for Scientific Research) Grant Numbers JP19J20990, JP18K19830, JP15H01715, JP24104005, and JP20H00618.

Open Access This article is licensed under a Creative Commons Attribution 4.0 International License, which permits use, sharing, adaptation, distribution and reproduction in any medium or format, as long as you give appropriate credit to the original author(s) and the source, provide a link to the Creative Commons licence, and indicate if changes were made. The images or other third party material in this article are included in the article's Creative Commons licence, unless indicated otherwise in a credit line to the material. If material is not included in the article's Creative Commons licence and your intended use is not permitted by statutory regulation or exceeds the permitted use, you will need to obtain permission directly from the copyright holder. To view a copy of this licence, visit http://creativecommons.org/licen ses/by/4.0/.

\section{References}

1. Prigogine, I., Lefever, R.: Theory of dissipative structures. Synergetics, pp. 124-135. Vieweg+ Teubner Verlag, Wiesbaden (1973)

2. Wolpert, L., Cheryll, T., Arias, A.M.: Principles of Development. Oxford University Press, USA (2015)

3. Murray II, J.D.: Spatial Models and Biomedical Applications. Springer, Berlin (2003)

4. Turing, A.M.: The chemical theory of morphogenesis. Philos. Trans. R. Soc. B237, 37-72 (1952)

5. Kondo, S., Asai, R.: A reaction-diffusion wave on the skin of the marine angelfish Pomacanthus. Nature 376(6543), 765 (1995)

6. Kondo, S., Miura, T.: Reaction-diffusion model as a framework for understanding biological pattern formation. Science 329(5999), 1616-1620 (2010)

7. Meinhardt, H.: The Algorithmic Beauty of Sea Shells. Springer, Berlin (2009)

8. Belousov, B. P. Ref. Radiats. Med. (1958). Medgiz, Moscow, 145. (1959).

9. Zhabotinsky, A.M.: Periodical oxidation of malonic acid in solution (a study of the Belousov reaction kinetics). Biofizika 9, 306-311 (1964)

10. Sirimungkala, A., Försterling, H.D., Dlask, V., Field, R.J.: Bromination reactions important in the mechanism of the Belousov-Zhabotinsky system. J. Phys. Chem. A 103(8), 1038-1043 (1999)

11. Bánsági Jr., T., Leda, M., Toiya, M., Zhabotinsky, A.M., Epstein, I.R.: High-frequency oscillations in the Belousov-Zhabotinsky reaction. J. Phys. Chem. A 113(19), 5644-5648 (2009)

12. Watson, J.D., Francis, H.C.C.: "The Structure of DNA". Cold Spring Harbor Symposia on Quantitative Biology, vol. 18. Cold Spring Harbor Laboratory Press, New York (1953)

13. Dirks, R.M., Pierce, N.A.: A partition function algorithm for nucleic acid secondary structure including pseudoknots. J. Comput. Chem. 24(13), 1664-1677 (2003)

14. SantaLucia, J., Allawi, H.T., Seneviratne, P.A.: Improved nearest-neighbor parameters for predicting DNA duplex stability. Biochemistry 35(11), 3555-3562 (1996)

15. SantaLucia, J.: A unified view of polymer, dumbbell, and oligonucleotide DNA nearest-neighbor thermodynamics. Proc. Natl. Acad. Sci. 95(4), 1460-1465 (1998)

16. Zhang, D.Y., Winfree, E.: Control of DNA strand displacement kinetics using toehold exchange. J. Am. Chem. Soc. 131(47), 17303-17314 (2009)

17. Dirks, R.M., Bois, J.S., Schaeffer, J.M., Winfree, E., Pierce, N.A.: Thermodynamic analysis of interacting nucleic acid strands. SIAM Rev. 49, 65-88 (2007) 
18. Dirks, R.M., Pierce, N.A.: An algorithm for computing nucleic acid base-pairing probabilities including pseudoknots. J. Comput. Chem. 25, 1295-1304 (2004)

19. Dirks, R.M., Pierce, N.A.: A partition function algorithm for nucleic acid secondary structure including pseudoknots. J. Comput. Chem. 24, 1664-1677 (2003)

20. Zhang, D.Y., Georg, S.: Dynamic DNA nanotechnology using strand-displacement reactions. Nat. Chem. 32, 103 (2011)

21. Okamoto, A., Tanaka, K., Saito, I.: DNA logic gates. J. Am. Chem. Soc. 126(30), 9458-9463 (2004)

22. Chen, X.: Expanding the rule set of DNA circuitry with associative toehold activation. J. Am. Chem. Soc. 134(1), 263-271 (2011)

23. Srinivas, N., Parkin, J., Seelig, G., Winfree, E., Soloveichik, D.: Enzyme-free nucleic acid dynamical systems. Science 358(eaal2052), 2052 (2017)

24. Seelig, G., Soloveichik, D., Zhang, D.Y., Winfree, E.: Enzyme-free nucleic acid logic circuits. Science 314(5805), 1585-1588 (2006)

25. Soloveichik, D., Seelig, G., Winfree, E.: Proc. Natl. Acad. Sci. U. S. A. 107(12), 5393-5398 (2010)

26. Baccouche, A., Montagne, K., Padirac, A., Fujii, T., Rondelez, Y.: Methods 67(2), 234-249 (2014)

27. Gines, G., Zadorin, A.S., Galas, J.C., Fujii, T., Estevez-Torres, A., Rondelez, Y.: Microscopic agents programmed by DNA circuits. Nat. Nanotechnol. 12(4), 351 (2017)

28. Genot, A.J., Baccouche, A., Sieskind, R., Aubert-Kato, N., Bredeche, N., Bartolo, J.F., Rondelez, Y.: High-resolution mapping of bifurcations in nonlinear biochemical circuits. Nat. Chem. 8(8), 760 (2016)

29. Scalise, D., Schulman, R.: Designing modular reaction-diffusion programs for complex pattern formation. Technology 2(01), 55-66 (2014)

30. Markham, N.R., Zuker, M.: DINAMelt web server for nucleic acid melting prediction. Nucleic Acids Res. 33(suppl_2), W577-W581 (2005)

31. Zadeh, J.N., Steenberg, C.D., Bois, J.S., Wolfe, B.R., Pierce, M.B., Khan, A.R., Pierce, N.A.: NUPACK: analysis and design of nucleic acid systems. J. Comput. Chem. 32(1), 170-173 (2011)

32. Lakin, M.R., Youssef, S., Polo, F., Emmott, S., Phillips, A.: Visual DSD: a design and analysis tool for DNA strand displacement systems. Bioinformatics 27(22), 3211-3213 (2011)

33. Dalchau, N., Seelig, G., Phillips, A.: Computational design of reaction-diffusion patterns using DNA-based chemical reaction networks. International Workshop on DNA-Based Computers, pp. 84-99. Springer, Cham (2014)

34. Tinland, B., Pluen, A., Sturm, J., Weill, G.: Persistence length of single-stranded DNA. Macromolecules 30(19), 5763-5765 (1997)

35. Chirieleison, S.M., Allen, P.B., Simpson, Z.B., Ellington, A.D., Chen, X.: Pattern transformation with DNA circuits. Nat. Chem. 5(12), 1000 (2013)

36. Zenk, J., Scalise, D., Wang, K., Dorsey, P., Fern, J., Cruz, A., Schulman, R.: Stable DNA-based reaction-diffusion patterns. RSC Adv. 7(29), 18032-18040 (2017)

37. Hosoya, T., Kawamata, I., Shin-ichiro, M.N., Murata, S.: Pattern formation on discrete gel matrix based on DNA computing. New Gener. Comput. 37(1), 97-111 (2019)

38. Abe, K., Kawamata, I., Shin-ichiro, M.N., Murata, S.: Programmable reactions and diffusion using DNA for pattern formation in hydrogel medium. Mol. Syst. Des. Eng. 20, 20 (2019)

39. Zadorin, A.S., Rondelez, Y., Gines, G., Dilhas, V., Urtel, G., Zambrano, A., Estévez-Torres, A.: Synthesis and materialization of a reaction-diffusion French flag pattern. Nat. Chem. 9(10), 990 (2017)

40. Wolpert, L.: Positional information and the spatial pattern of cellular differentiation. J. Theor. Biol. 25(1), 1-47 (1969)

41. Padirac, A., Fujii, T., Estévez-Torres, A., Rondelez, Y.: Spatial waves in synthetic biochemical networks. J. Am. Chem. Soc. 135(39), 14586-14592 (2013)

42. Goel, N.S., Maitra, S.C., Montroll, E.W.: On the Volterra and other nonlinear models of interacting populations. Rev. Mod. Phys. 43(2), 231 (1971)

43. Hagiya, M., Wang, S., Kawamata, I., Murata, S., Isokawa, T., Peper, F., Imai, K.: On DNA-based gellular automata. Lect. Notes Comput. Sci. 8553, 177 (2014)

44. Takabatake, F., Kawamata, I., Sugawara, K., Murata, S.: Discretization of chemical reactions in a periodic cellular space. New Gener. Comput. 35(3), 213-223 (2017)

45. Kawamata, I., Hosoya, T., Takabatake, F., Sugawara, K., Nomura, S.I., Isokawa, T., Peper, F., Hagiya, M., Murata, S.: Pattern formation and computation by autonomous chemical reaction diffusion model inspired by cellular automata. In: The Fourth International Symposium on Computing and Networking, pp. 215-221 (2016). 
46. Rodjanapanyakul, T., Takabatake, F., Abe, K., Kawamata, I., Nomura, S.M., Murata, S.: Diffusion modulation of DNA by toehold exchange. Phys. Rev. E 97(5), 052617 (2018)

47. Urtel, G., Estevez-Torres, A., Galas, J.C.: DNA-based long-lived reaction-diffusion patterning in a host hydrogel. Soft Matter 20, 20 (2019)

48. Akam, M.: The molecular basis for metameric pattern in the Drosophila embryo. Development 101(1), 1-22 (1987)

49. St Johnston, D., Nüsslein-Volhard, C.: The origin of pattern and polarity in the Drosophila embryo. Cell 68(2), 201-219 (1992)

50. Isalan, M., Lemerle, C., Serrano, L.: Engineering gene networks to emulate Drosophila embryonic pattern formation. PLoS Biol. 3, 3 (2005)

51. Weber, W., Fussenegger, M.: Engineering of synthetic mammalian gene networks. Chem. Biol. 16(3), 287-297 (2009)

52. Lin, D.C., Yurke, B., Langrana, N.A.: Mechanical properties of a reversible, DNA-crosslinked polyacrylamide hydrogel. J. Biomech. Eng. 126(1), 104-110 (2004)

53. Zhu, Z., Wu, C., Liu, H., Zou, Y., Zhang, X., Kang, H., Tan, W.: An aptamer cross-linked hydrogel as a colorimetric platform for visual detection. Angew. Chem. Int. Ed. 49(6), 1052-1056 (2010)

54. Murakami, Y., Maeda, M.: DNA-responsive hydrogels that can shrink or swell. Biomacromol 6(6), 2927-2929 (2005)

55. Cangialosi, A., Yoon, C., Liu, J., Huang, Q., Guo, J., Nguyen, T.D., Schulman, R.: DNA sequencedirected shape change of photopatterned hydrogels via high-degree swelling. Science 357(6356), 1126-1130 (2017)

56. Dirks, R.M., Pierce, N.A.: Triggered amplification by hybridization chain reaction. Proc. Natl. Acad. Sci. 101(43), 15275-15278 (2004)

57. Boekhoven, J., Poolman, J.M., Maity, C., Li, F., Van Der Mee, L., Minkenberg, C.B., Eelkema, R.: Catalytic control over supramolecular gel formation. Nat. Chem. 5(5), 433 (2013)

58. Lovrak, M., Hendriksen, W.E., Maity, C., Mytnyk, S., van Steijn, V., Eelkema, R., van Esch, J.H.: Free-standing supramolecular hydrogel objects by reaction-diffusion. Nat. Commun. 8, 15317 (2017)

59. Lovrak, M., Hendriksen, W.E., Kreutzer, M.T., van Steijn, V., Eelkema, R., van Esch, J.H.: Control over the formation of supramolecular material objects using reaction-diffusion. Soft Matter 20, 20 (2019)

60. Kandatsu, D., Cervantes-Salguero, K., Kawamata, I., Hamada, S., Nomura, S.I.M., Fujimoto, K., Murata, S.: Reversible gel-sol transition of a photo-responsive DNA gel. ChemBioChem 17(12), 1118-1121 (2016)

61. Murata, S., Konagaya, A., Kobayashi, S., Saito, H., Hagiya, M.: Molecular robotics: a new paradigm for artifacts. New Gener. Comput. 31(1), 27-45 (2013)

Publisher's Note Springer Nature remains neutral with regard to jurisdictional claims in published maps and institutional affiliations. 